\title{
Study on Migration of Two Triazine Type Ultraviolet Absorbents from Polypropylene Packaging Materials to Food Simulant
}

\author{
Yue Qiu ${ }^{1,2 *}$, Yan Ruan ${ }^{1}$, and Genrong $\mathrm{Li}^{1}$ \\ ${ }^{1}$ Chongqing Academy of Metrology and Quality Inspection, Chongqing, China \\ ${ }^{2}$ College of Chemistry and Chemical Engineering, Chongqing University, Chongqing, China
}

\begin{abstract}
The migration rules of two triazine type of UV absorbents (UV-1577 and UV-1579) from polypropylene (PP) food contact materials to food simulant were investigated. A rapid detection method of UV-1577 and UV-1579 in PP food contact materials was established by ultra-performance convergence chromatography $\left(\mathrm{UPC}^{2}\right)$. The optimal extraction method of the initial solution was determined, and the pretreatment methods of two UV absorbents in the liquid simulants were optimized. Experiment results showed that the interaction effects between the two UV absorbers and the liquid simulants were weak, and the migration behavior was the most obvious in the fatty simulant of isooctane. At the same temperature, the migration amount of both UV-1577 and UV-1579 increased with the extension of time, and UV-1577 was more prone to migration than UV-1579. Within a certain range, the migration amount of the two UV absorbents increased significantly with the increase of the migration temperature. The higher the temperature was, the shorter the time required to reach the equilibrium.
\end{abstract}

\section{Introduction}

Polypropylene (PP) food grade plastic is widely used in the production of a variety of food packaging containers due to the good characteristics concluding light weight, impact resistance and high temperature resistance. PP food contact materials are easy to oxidize and yellowing under prolonged light conditions. Therefore, UV absorbents are often added in the production process to reduce UV radiation damage and degradation of PP materials[1-3].

As a common food packaging additive, UV absorbents are mainly divided into salicylate, benzotriazole and triazine. Triazine UV-absorbent is a new kind of UV-absorbent with light color, high UV absorbance and less dosage. UV absorbents may pose a threat to human health by migrating from food contact materials to food[4-7].

In recent years, a large number of studies have focused on the migration of benzotriazole UVabsorbents from various plastic food contact materials [810]. However, the migration of triazine UV-absorbents from PP materials to food simulants has not received widespread attention. In this paper, the detection method and migration rules of the two widely used triazine UV absorbents (UV-1577 and UV-1579) in PP food contact materials were studied, which could provide technical reference for relevant departments to supervise the safety of plastic food contact materials.

\section{EXPERIMENTAL METHODS}

\subsection{Reagents and Instruments}

UV-1577 and UV-1579 were supplied from Dr. Ehrenstorfer (Augsburg, Germany). HPLC grade methanol, isopropanol and acetonitrile were purchased from Merck (Darmstadt, Germany). The detection method was performed on ACQUITY UPC ${ }^{2}$ ultra performance convergence chromatography (Waters, USA). N-evap-112 nitrogen blower (Organomation, USA) and Bilon-2000 CT ultrasonic cleaner (Bilang, Shanghai) were used in the preprocessing.

\subsection{Chromatographic conditions}

Column: ACQUITY UPC ${ }^{2}$ HSS C18 SB column (150 $\mathrm{mm} \times 30 \mathrm{~mm}, 1.8 \mu \mathrm{m}$ ); Column temperature: $35^{\circ} \mathrm{C}$; Mobile phase: supercritical $\mathrm{CO}_{2}$-methanol; the elution gradient was (eluent $\mathrm{A}: \mathrm{CO}_{2}$; eluent $\mathrm{B}$ : isopropanol): 0-1 min, $8 \% \mathrm{~B}-8.5 \% \mathrm{~B} ; 1-1.8 \mathrm{~min}, 8.5 \% \mathrm{~B}-9 \% \mathrm{~B} ; 1.8-2 \mathrm{~min}$, $9 \% \mathrm{~B}-25 \% \mathrm{~B}$; $2-4$ min, $25 \% \mathrm{~B}$; $4-4.5$ min, 25\%B-8.5\%B.

\subsection{Preparation of standards}

The standard substance $(1.0 \mathrm{mg})$ was accurately weighed into a $10 \mathrm{~mL}$ volumetric flask and dissolved in methanol and volumetric to prepare a standard solution with a concentration of $100 \mathrm{mg} / \mathrm{L}$. The standard working

\footnotetext{
*Corresponding author: qiuyuecqu@163.com
} 
solution of $0.1-100.0 \mathrm{mg} / \mathrm{L}$ was prepared by stepwise dilution method.

\subsection{Determination of initial solubility of UV absorbents}

The samples were cut into pieces and accurately weighed $1.0 \mathrm{~g}$ to be tested. Then the sample was extracted by ultrasonic treatment with methanol, n-hexane, ethyl acetate or chloroform. Afterwards, the extracted solution was dried with nitrogen flow at $45^{\circ} \mathrm{C}$ and diluted to 1.0 $\mathrm{mL}$ with methanol and filter with $0.22 \mu \mathrm{m}$ organic membrane for $\mathrm{UPC}^{2}$ analysis.

\subsection{Migration experiment}

PP samples were immersed in food simulants at temperature of $4,20,40,60$ and $80^{\circ} \mathrm{C}$, and taking samples at the time points of $2,4,8,12,24,48,120$ and $240 \mathrm{~h}$, respectively.

\section{Results and analysis}

\subsection{Linearity, LODs and LOQs}

The samples were respectively injected at a concentration of $0.1,1.0,5.0,20.0,50.0,80.0$ and 100.0 $\mathrm{mg} / \mathrm{L}$ and quantified by external standard method. The chromatographic peak area $\mathrm{Y}$ of each substance was linearly to the mass concentration $X$. Results showed that the correlation coefficients of the two UV absorbent were greater than 0.999 . The mixed standard solutions with different concentrations were added in the blank food simulants without UV absorbents. The detection limits (LODs) were calculated according to three times of signal-to-noise ratio $(\mathrm{S} / \mathrm{N}=3)$. The LOD of the two UV absorbents in the aqueous simulants (water, 3\% acetic acid and $10 \%$ ethanol) was $0.15 \mathrm{mg} / \mathrm{L}$, and the LOD of the two UV absorbents in the isooctane simulants was $0.05 \mathrm{mg} / \mathrm{L}$.

\subsection{Determination of initial solubility of UV absorbents}

In order to detect the migration of UV absorbent, the initial content of UV absorbents in PP samples should be analyzed first, the dissolution of UV-1577 and UV-1579 in PP sample was determined by soxhlet extraction, ultrasonic extraction and immersion extraction respectively. The results (Fig. 1) showed that the extraction efficiency of ultrasonic extraction method was the highest, while that of immersion extraction was the lowest. Compared with four extraction solvents, the initial dissolution amount of the two UV absorbents extracted with chloroform was the largest. The reason may be due to the destruction of the molecular structure of part of PP by chloroform, making the UV absorbents easier to be extracted after exposure to the outside. Therefore, ultrasonic extraction method with chloroform

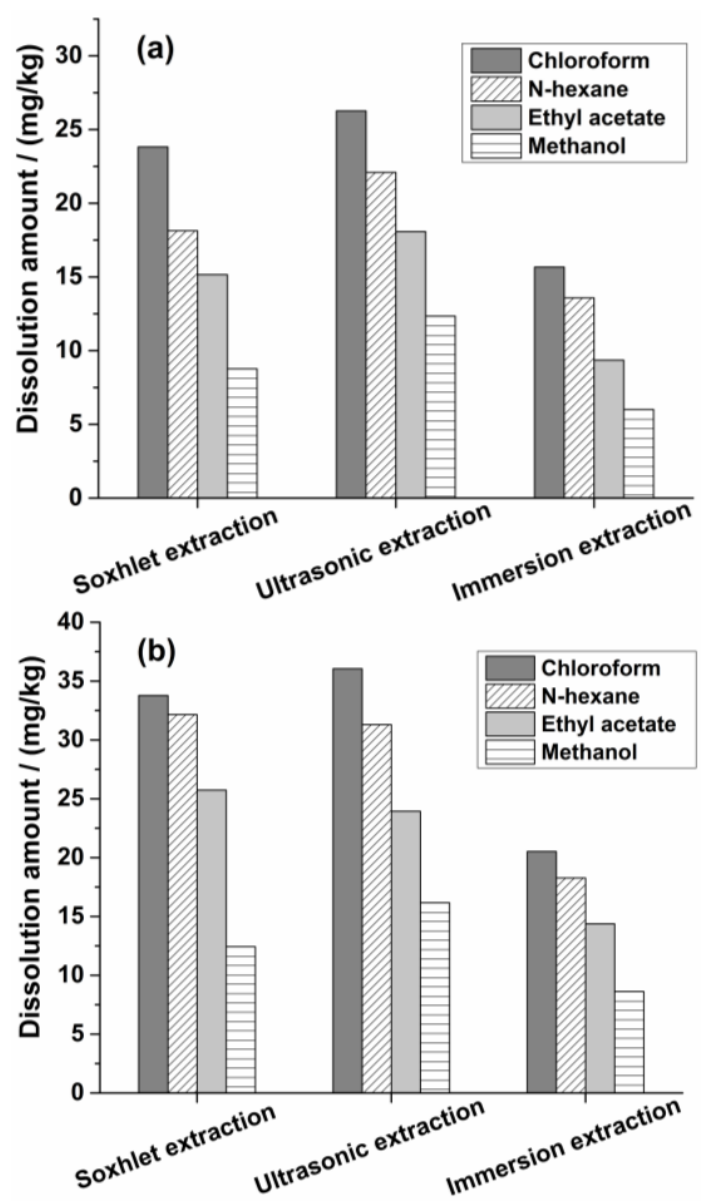

Fig. 1 Initial dissolution amount of (a) UV-1577 and (b) UV1579 under different extraction methods and solvent conditions

as solvent was used as the best extraction method for extraction of initial dissolution.

\subsection{Selection of extraction solvent for aqueous food simulants}

Three blank water-based simulants (water, 3\% acetic acid, and $10 \%$ ethanol) were added with UV standards at high, medium, low concentrations, respectively. Toluene, ethyl acetate, n-hexane and dichloromethane were used as extraction solvent. As shown in Fig. 2, the extraction efficiency of dichloromethane was the best, and the recoveries of the two UV absorbents in different simulants was higher than $85.0 \%$.

\subsection{The influence of simulants on UV absorbents migration}

The PP microwave box sample was immersed in distilled water, $3 \%$ acetic acid, $10 \%$ ethanol, $30 \%$ ethanol, $70 \%$ ethanol and isooctane at $40^{\circ} \mathrm{C}$ for $24 \mathrm{~h}$ to investigate the effect of simulants on UV absorbent migration. The results (Table 1) showed that UV-1577 and UV-1579 did not dissolve out in pure water matrix and acidic food simulant. However, they were observed in the alcohol and fatty simulants. Compared with the alcohol, the migration behavior of the two UV absorbents in isooctane was the most obvious. This was 


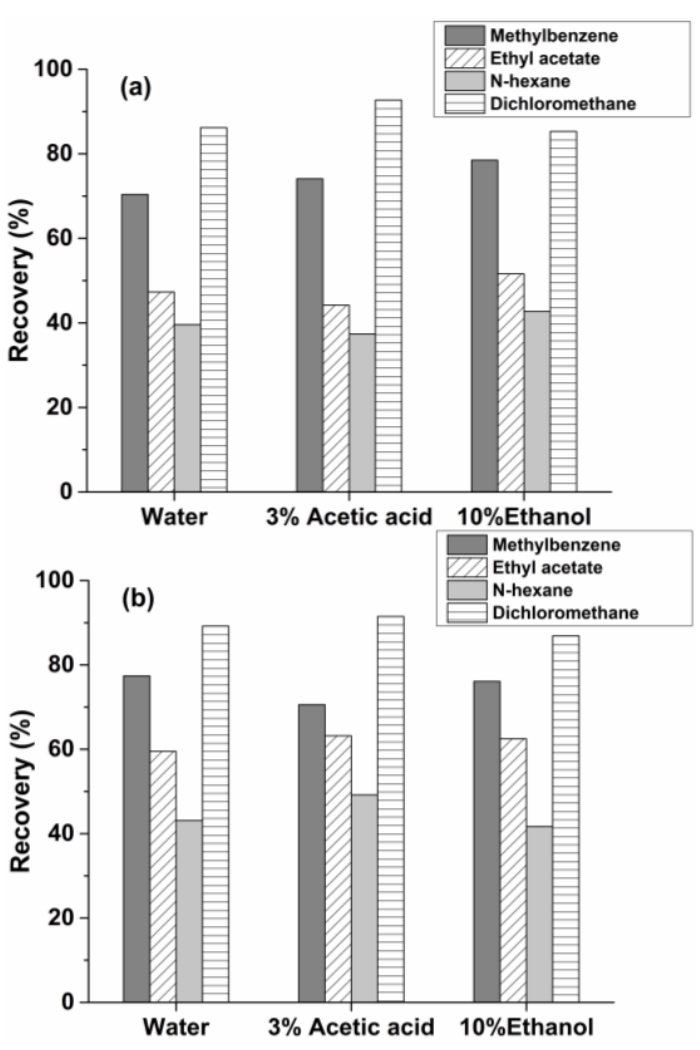

Fig.2 Effects of extraction solvent on the recoveries of (a) UV1577 and (b) UV-1579 in different simulants

due to that UV-1577 and UV-1579 were oleophilic substances with high steric hindrances, which hindered the interaction effect between $\mathrm{N}$ atoms and hydroxyl groups on the triazine ring and polar simulant.

Table 1. The migration of two UV absorbents in different simulants $\left(40^{\circ} \mathrm{C}, 24 \mathrm{~h}\right)$

\begin{tabular}{ccccccc}
\hline Simulants & $\mathrm{W}$ & $\begin{array}{c}3 \% \\
\mathrm{~A}\end{array}$ & $10 \% \mathrm{E}$ & $30 \% \mathrm{E}$ & $70 \% \mathrm{E}$ & $\mathrm{I}$ \\
\hline $\begin{array}{c}\mathrm{UV}-1577 \\
/(\mathrm{mg} / \mathrm{kg})\end{array}$ & $\mathrm{N}$ & $\mathrm{N}$ & 0.165 & 0.517 & 1.926 & 4.017 \\
$\mathrm{UV}-1579$ & $\mathrm{~N}$ & $\mathrm{~N}$ & 0.226 & 0.581 & 1.546 & 2.431 \\
\hline$/(\mathrm{mg} / \mathrm{kg})$ & $\mathrm{N}$ & & & & \\
\hline
\end{tabular}

$\mathrm{N}$ : below the LOD;

W: water;

A: acid;

E: ethanol;

$\mathrm{I}$ : isooctane.

\subsection{The influence of migration temperature and time on UV absorbents migration}

Subsequently, the effects of migration temperature and time on UV-1577 and UV-1579 migration in isooctane simulant were investigated. As shown in Fig. 3, the migration amount of UV-1577 in isooctane was greater than that of UV-1579 under the same migration conditons. At the same temperature, the migration of the two UV absorbents increased with the extension of the
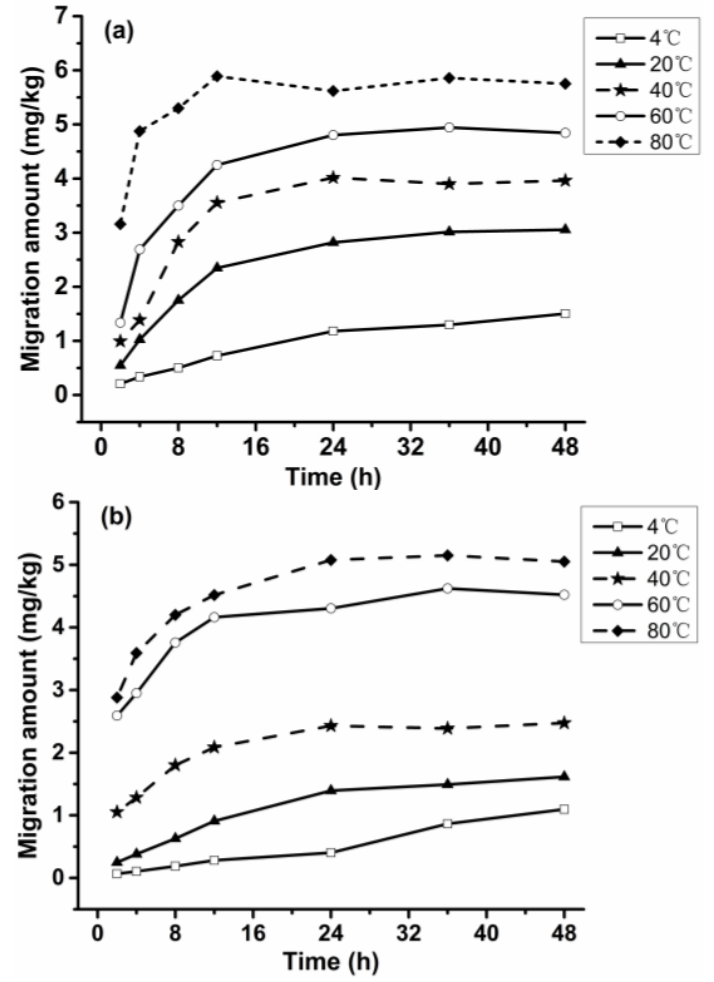

Fig.3 Effects of migration temperature and time on migration of (a) UV-1577 and (b) UV-1579

migration time. With the increase of the migration temperature, the time for UV-1577 and UV-1579 to reach the migration equilibrium decreased and the migration amount increased.

\section{Conclusion}

In this paper, $\mathrm{UPC}^{2}$ was used to establish a method for the detection of UV-1577 and UV-1579 in polypropylene (PP) food contact materials. The experiment investigated the extraction solvents of two UV absorbers. Experiment results showed that dichloromethane was the best extraction solvent. By studying the migration rules of UV-1577 and UV-1579 in different food simulants, it was found that the interaction effect between the two UV absorbents and aqueous simulants was weak, and the migration behavior was the most obvious in isooctane. At the same temperature, the migration of UV-1577 and UV-1579 increased with the extension of time, and UV-1577 was more likely to migrate than UV-1579. Within a certain range, with the increase of the migration temperature, the migration speed and the migration amount of the two UV absorbents increased significantly, and the time to reach the migration equilibrium was shortened.

\section{References}

1. D. Baran, A. Balan, T. Stubhan, T. Ameri, L. Toppare, C. Brabec, Synth. Met. 162, 2047 (2012)

2. K. Pei, Z.H. Cui, W.G. Chen, J. Mol. Struct. Vol. 1032, 100 (2013) 
3. S.S. Choi, M.J. Song, Rapid Commun. Mass Spectrom. 22, 2580 (2008),

4. J.W. Kin, T. Isobe, B.R. Ramaswamy, K.H.Chang, A. Amano, T.M. Miller, F.P. Siringan, S. Tanabe, Chemosphere 85, 751 (2011)

5. K. Fent, G. Chew, J. Li, E. Gomez, Sci. Total Environ. 482, 125 (2014)

6. J.W. Kin, K.H. Chang, T. Isobe, S. Tanabe, J. Toxicol. Sci. 36, 247 (2011)

7. H. Nakata, S. Murata, J. Filatreau, Environ. Sci. Tech. 43, 6920 (2009)

8. C. Li, Y. LI, Z. Chen, F. Liang, X. Chen, S. Wu, Y. Li, X. Sun, Food Anal. Methods 7, 1755 (2014)

9. J. Wang, X. Xiao, T. Chen, T. Liu, H. Tao, J. He, J. Chromatogr. A 1451, 58 (2016)

10. W.A.C. Anderson, L. Castle, Food Addit. Contam. 20, 607 (2003) 